\title{
Accurate UWB Radar Three-Dimensional Imaging Algorithm for a Complex Boundary Without Range Point Connections
}

\author{
Shouhei Kidera, Associate Member, IEEE, Takuya Sakamoto, Member, IEEE, and Toru Sato, Member, IEEE
}

\begin{abstract}
Ultrawide-band pulse radars have immeasurable potential for a high-range-resolution imaging in the near field and can be used for noncontact measurement of industrial products with specular or precision surfaces, such as reflector antenna or aircraft fuselage, or identifying and locating the human body in security systems. In our previous work, we developed a stable and high-speed 3-D imaging algorithm, Envelope, which is based on the principle that a target boundary can be expressed as inner or outer envelopes of spheres, which are determined using antenna location and observed ranges. Although Envelope produces a high-resolution image for a simple shape target that may include edges, it requires an exact connection for observed ranges to maintain the imaging quality. For complex shapes or multiple targets, this connection becomes a difficult task because each antenna receives multiple echoes from many scattering points on the target surface. This paper proposes a novel imaging algorithm without range point connection to accomplish high-quality and flexible 3-D imaging for various target shapes. The algorithm uses an accurate estimation for the direction of arrival using signal amplitudes and realizes direct mapping from observed ranges to target points. Several comparative studies of conventional algorithms clarify that our proposed method accomplishes accurate and reliable 3-D imaging even for complex or multiple boundaries.
\end{abstract}

Index Terms-Accurate and stable 3-D imaging, complex boundary, direction of arrival (DOA) estimation, multiple targets, range point connection, ultrawide-band (UWB) pulse radars.

\section{INTRODUCTION}

$\mathbf{U}$ LTRAWIDE-BAND pulse radars have great potential for use in superresolution imaging, which is required in nearfield sensing applications such as target identification and selflocation by robots or automobiles. They can be applied to surveillance or security systems for intruder detection or aged care, where an optical camera has the serious problem of intruding on privacy in bathrooms or living rooms. They are also suitable for noncontact measurement of reflector antennas or aircraft bodies that have high-precision and specular surfaces, where an extremely accurate surface measurement is

Manuscript received June 25, 2008; revised June 5, 2009 and September 4, 2009. First published January 15, 2010; current version published March 24, 2010. This work was supported in part by the Grant-in-Aid for Scientific Research (A) under Grant 17206044 and in part by the Grant-in-Aid for Japan Society for the Promotion of Science (JSPS) Fellows under Grant 19-497.

$\mathrm{S}$. Kidera is with the Department of Electronic Engineering, Graduate School of Electro-Communications, The University of Electro-Communications, Tokyo 182-8585, Japan (e-mail: kidera@ee.uec.ac.jp).

T. Sakamoto and T. Sato are with the Department of Communications and Computer Engineering, Graduate School of Informatics, Kyoto University, Kyoto 606-8501, Japan (e-mail: t-sakamo@i.kyoto-u.ac.jp; tsato@kuee. kyoto-u.ac.jp).

Digital Object Identifier 10.1109/TGRS.2009.2036909 required for the product inspection. Although various kinds of radar algorithms based on data synthesis have been proposed, such as synthetic aperture radar (SAR) [1], time-reversal algorithms [2], [3], range migration methods [4]-[7], and other optimization algorithms [8]-[11], they all require intensive computation and are hardly applicable to the aforementioned applications. Contrarily, the high-speed 3-D imaging algorithm SEABED achieves direct and nonparametric imaging based on reversible transforms, namely, boundary scattering transform (BST) and inverse BST (IBST), between the time delay and target boundary [12]-[15]. However, imaging using SEABED becomes unstable for noisy data because the range derivative in BST can enhance the fluctuation of small range errors. To produce a more stable image, we have already proposed a realtime 3-D imaging algorithm named Envelope [16], [17]. This method uses an envelope of spheres, which are determined with antenna locations and observed ranges, to create a stable image without requiring derivative operations. It has been confirmed that this method robustly reconstructs a high-resolution 3-D image for objects of simple shape, including those with edges when combined with the range compensation method termed spectrum offset correction [17]. Moreover, an extended algorithm has been published, based on this method and which can extract an accurate breast surface for cancer detection [18]. In addition, the ultrasound imaging for airborne objects has been developed, which is based on the similar principle that a scattering point must be tangent to a sphere or ellipsoid determined by an antenna location and observed range [19]. However, the image obtained with Envelope becomes inaccurate for complex boundaries because it requires an appropriate range connection. For a complex surface, this connection is often difficult because each antenna observes multiple echoes, and there are too many candidates for the connections. Hantscher et al. [20] have developed an iterative wavefront extraction method for multiple targets, which recursively subtracts scattered waveforms to resolve multiple echoes. However, once the range connections fail, there is nonnegligible inaccuracy in the images obtained by these conventional algorithms. A global optimization algorithm based on waveform matching has been developed [21], yet it still requires a long calculation time. For the ground penetrating radar applications, Windsor et al. [22] have proposed the efficient connection algorithm for observed range points, which uses a cross-correlation of received signals between adjacent antenna locations. In any event, all conventional algorithms that are specific to either SEABED or Envelope have a substantial 


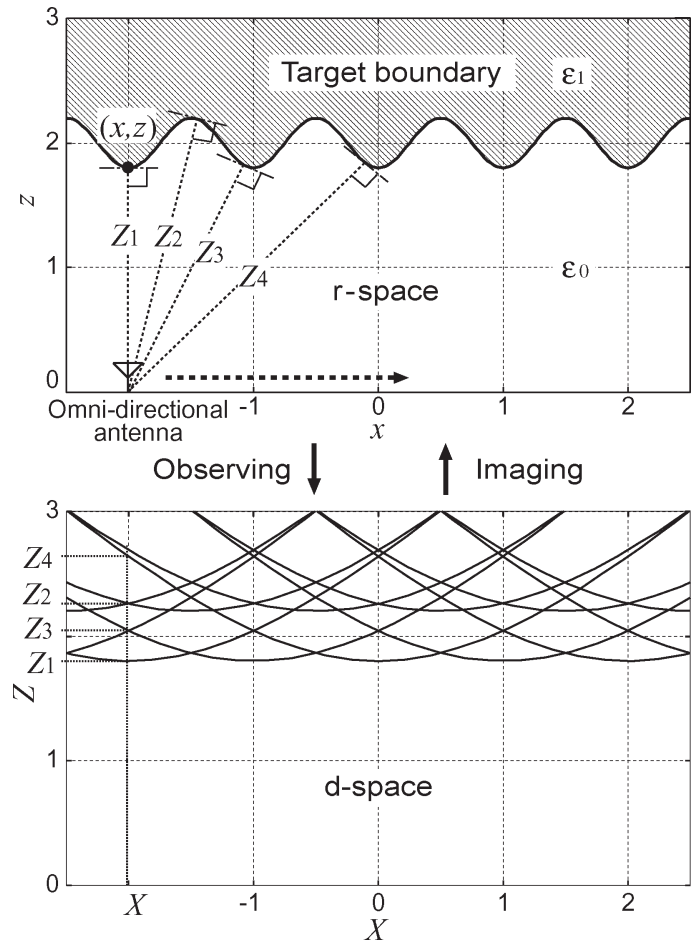

Fig. 1. Relationship between (upper) target boundary in r-space and (lower) quasi-wavefront in d-space.

problem in that they are extremely sensitive to inappropriate connections of range points.

This paper proposes a novel algorithm based on direct group mapping from observed ranges to target boundary points without having range connection. This algorithm involves an accurate estimation for the direction of arrival (DOA) using signal amplitudes, which eliminates the range connecting procedure. The idea is based on a simple principle, yet it remarkably enhances an accuracy even in complex boundary extraction. First, the algorithm for a 2-D model is presented for simplicity, and it is then extended for a 3-D model. This paper also presents comparative studies using several conventional algorithms, such as SAR and Fourier transform. The numerical simulations indicate that our proposed method has a significant advantage in accurate imaging even for complex shape or multiple targets. Although this paper assumes a monostatic radar system, it is readily extended to the bistatic model. The weakness of the monostatic radar is that the target surface estimated is limited compared with a bistatic radar because strong echoes are observed only from the surface perpendicular to the the line of sight. Contrarily, the advantage is that a monostatic radar system can be relatively compact, while a bistatic radar system [23] uses a large-scale antenna array.

\section{2-D PROBLEM}

\section{A. System Model and Data Processing}

The upper diagram in Fig. 1 shows the system model. It assumes that the target has an arbitrary shape with a clear boundary and that the propagation speed of the radio wave is a known constant. It assumes the monostatic radar, and an

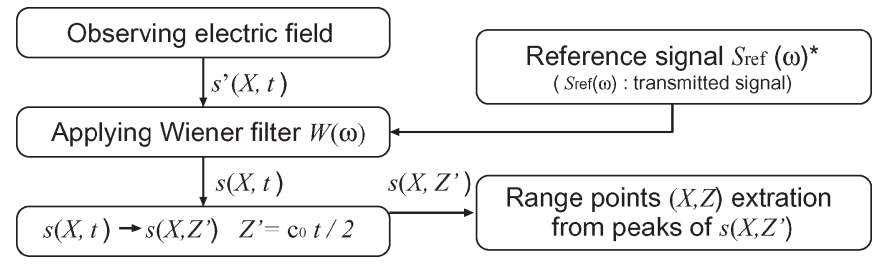

Fig. 2. Diagram for the data processing.

omnidirectional antenna is scanned along the $x$-axis. We use a monocycle pulse as the transmitting current. The r-space is defined as the real space in which the target and antenna are located, and it is expressed by the parameters $(x, z)$. The parameters are normalized by $\lambda$, which is the central wavelength of the pulse. We assume $z>0$ for simplicity.

$s^{\prime}(X, t)$ is defined as the electric field received at antenna location $(x, z)=(X, 0)$ at time $t . s(X, t)$ is calculated by applying the Wiener filter to $s^{\prime}(X, t)$ as

$$
s(X, t)=\int_{-\infty}^{\infty} W(\omega) S^{\prime}(X, \omega) e^{j \omega t} d \omega
$$

where $S^{\prime}(X, \omega)$ is the signal in the frequency domain of $S^{\prime}(X, t) . W(\omega)$ is defined as

$$
W(\omega)=\frac{S_{\mathrm{ref}}(\omega)^{*}}{(1-\eta) S_{0}^{2}+\eta\left|S_{\mathrm{ref}}(\omega)\right|^{2}} S_{0}
$$

where $\eta=1 /\left(1+(S / N)^{-1}\right)$ and $S_{\text {ref }}(\omega)$ is the reference signal in the frequency domain, which is the complex conjugate of that of the transmitted signal. $S_{0}$ is a constant for dimension consistency. This filter is an optimal mean square error linear filter for additive noises. With a high $S / N(\eta \simeq 1)$, it functions as an inverse filter, and while with a low $S / N(\eta \simeq 0)$, it functions as a matched filter. $s(X, t)$ is converted to $s\left(X, Z^{\prime}\right)$ using the valuable conversion $Z^{\prime}=c_{0} t / 2 \lambda$, where $c_{0}$ is the speed of the radio wave. $(X, Z)$ is defined as the range point which is extracted from the peaks of $s\left(X, Z^{\prime}\right)$ as

$$
\begin{aligned}
\partial s\left(X, Z^{\prime}\right) / \partial Z^{\prime} & =0 \\
s\left(X, Z^{\prime}\right) & \geq \alpha \max _{Z^{\prime}} s\left(X, Z^{\prime}\right) .
\end{aligned}
$$

The parameter $\alpha \geq 0$ is empirically determined. The rational behind $\alpha>0$ is that the response of the Wiener filter to a single scattered wave is positive, because it has almost the same phase relationship to the reference signal $S_{\text {ref }}(\omega)$. Fig. 2 shows the data processing diagram from the observed electric field $s^{\prime}(X, t)$ to the range points $(X, Z)$.

The $\mathrm{d}$-space is defined as the space expressed by $(X, Z)$. Fig. 1 shows the relationship between $(x, z)$ in r-space and $(X, Z)$ in $\mathrm{d}$-space. The transform from $\mathrm{d}$-space to $\mathrm{r}$-space corresponds to the imaging, dealt with in this paper.

\section{B. Conventional Algorithms}

1) SAR Technique for Near-Field Imaging: The SAR technique is the most useful in radar imagery and is based on signal 


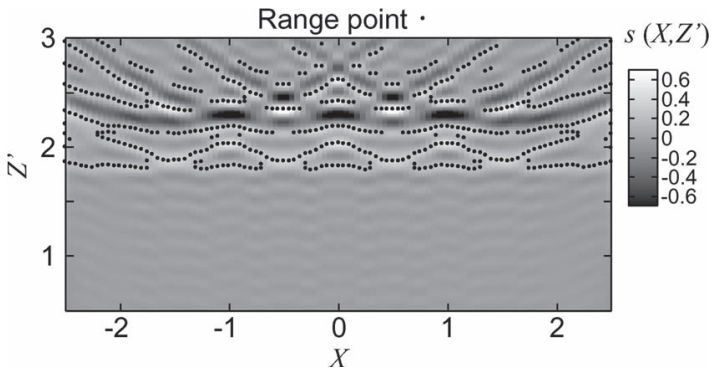

Fig. 3. Output of the Wiener filter and the extracted range points.

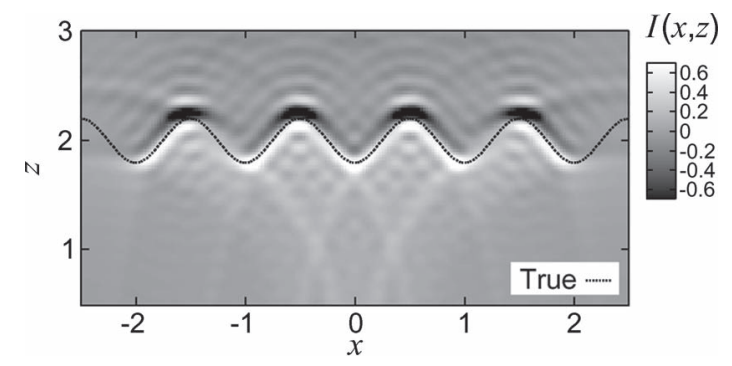

Fig. 4. Estimated image $I(x, z)$ with SAR.

synthesis [1]. In the near-field case, the distribution image $I(x, z)$ obtained using SAR is expressed as

$$
I(x, z)=\int_{-\infty}^{\infty} s\left(X, \sqrt{(x-X)^{2}+z^{2}}\right) d X .
$$

The target boundary can be extracted from its focused image $I(x, z)$. An example of applying SAR is presented here. The target boundary is assumed to be sinusoidal curve and is expressed as $z=2.0-0.2 \cos (2 \pi x)$. Fig. 3 shows the output of the Wiener filter and the extracted range points $(X, Z)$, where each signal is received at 101 locations for $-2.5 \leq X \leq 2.5$. Here, the finite-difference time domain (FDTD) is used for the calculation of the received signal $s^{\prime}(X, t)$. The conductivity and relative permittivity of the target are set to $1.0 \times 10^{6} \mathrm{~S} / \mathrm{m}$ and $\epsilon_{1}=1.0$, respectively. Moreover, the permittivity of air is $\epsilon_{0}=$ 1.0. From this figure, it is evident that there are some responses before the true time of arrival $(Z<1.8 \lambda)$, which are caused by an aliasing effect or cross-correlation response of the Wiener filter with characteristics of both the inverse and matched filters. Fig. 4 shows the estimated image with SAR, and the target boundary is highlighted. Although this method accomplishes a stable imaging even for a complex target boundary, it cannot clearly determine the location of target boundaries. Furthermore, this method requires searching operations for the entire assumed region $(x, z)$, and the calculation time is more than $60 \mathrm{~s}$ using a 2.8-GHz Xeon processor. To enhance the speed of the original SAR, a frequency-wavenumber $(\mathrm{F}-\mathrm{K})$ migration method has been developed [6], [7]. This algorithm is based on the aperture synthesis in the $\mathrm{F}-\mathrm{K}$ domains and calculates the migrated image $I_{\mathrm{FK}}(x, z)$ as

$$
I_{\mathrm{FK}}(x, z)=\int_{-\infty}^{\infty} \int_{-\infty}^{\infty} S\left(k_{X}, \omega\right) e^{j\left(k_{X} x+\sqrt{\frac{\omega^{2}}{c_{0}^{2}}-k_{X}^{2} z}\right)} d k_{X} d \omega
$$

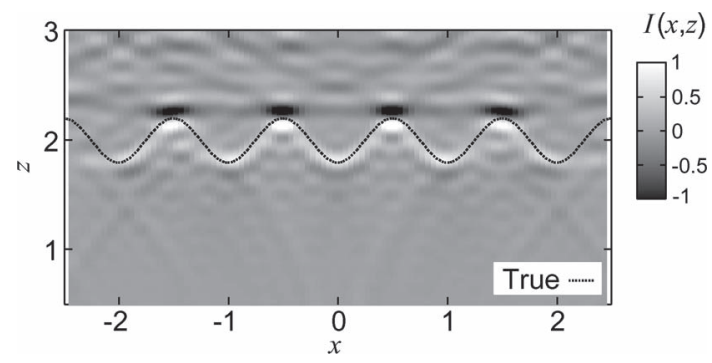

Fig. 5. Estimated image $I_{\mathrm{FK}}(x, z)$ with FK migration.

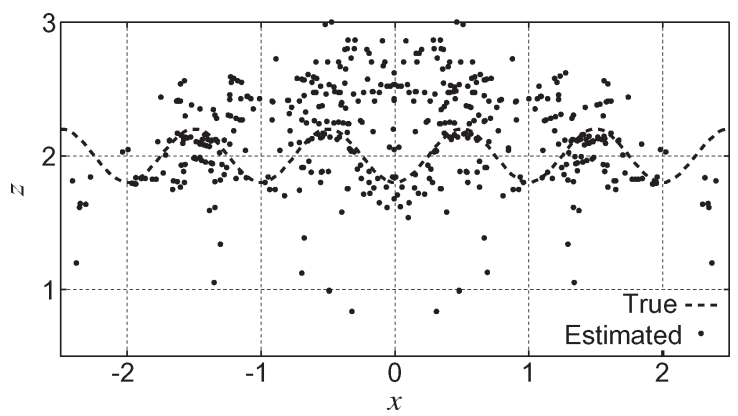

Fig. 6. Estimated image with SEABED.

where $S\left(k_{X}, \omega\right)$ is the output of the Wiener filter in the F-K domain, obtained by a 2-D Fourier transform of $s(X, t)$. Fig. 5 shows the resulting image $I_{\mathrm{FK}}(x, z)$. This algorithm makes use of the FFT algorithm, thereby increasing the imaging speed even for a massive amount of data. For the example image, the calculation time is $2.0 \mathrm{~s}$ using the same processor as before. Although the resulting image is almost the same as $I(x, z)$, there are small differences between the two images because the latter algorithm makes use of data interpolation in the $\mathrm{F}-\mathrm{K}$ domain, and this is often prone to error. Various algorithms, derived from the SAR method and aimed at resolution or speed enhancement [4], [5], have been proposed. These algorithms are, however, barely able to determine the clear surfaces of targets, particularly around the edges, since they are all based on focusing the received wavefield.

2) SEABED: We have already proposed a nonparametric imaging algorithm, known as SEABED, that drastically shortens the calculation time for target boundary extraction [13], [14]. For direct extraction of a clear target boundary, this algorithm connects the range points $(X, Z)$ for each $X$, creating what is called a quasi-wavefront. The reversible transform BST is then used between a target point in r-space and the quasiwavefront in d-space. IBST is expressed as

$$
\left.\begin{array}{l}
x=X-Z \partial Z / \partial X \\
z=Z \sqrt{1-(\partial Z / \partial X)^{2}}
\end{array}\right\}
$$

This transform provides a strict solution for the assumed inverse problem. The observed range and its derivative are directly transformed to the target boundary by IBST. We confirm that this method achieves high-speed and nonparametric imaging of a simple target. However, for a complex one, the image produced by SEABED is quite unstable, as shown in Fig. 6, where the same data as in Fig. 3 are used. This is because IBST uses the derivative of the quasi-wavefront. The small range 


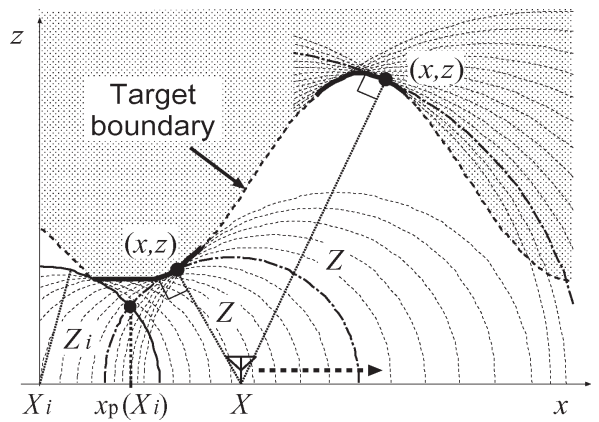

Fig. 7. Relationship between target boundary and envelopes of circles in 2-D problem.

fluctuations due to multiple interferences are enhanced by the range derivative $\partial Z / \partial X$ even if the condition $|\partial Z / \partial X| \leq 1$ is used. IBST also requires an appropriate range point connection to calculate an accurate derivative of quasi-wavefront. For the complicated range points shown in Fig. 3, this operation is extremely difficult. To overcome this difficulty, several methods for finding an appropriate range point connection have been developed, such as the iterative wavefront subtraction for multiple-target recognition [20] and using a global optimization algorithm based on waveform matching [21]. However, they all have a substantial problem in that if the range point connection fails, there is serious image distortion because an incorrect range connection causes large derivative errors.

3) Envelope: To suppress the instability due to fluctuation in the range derivative, a stable and rapid imaging algorithm Envelope has been developed [16]. This algorithm uses the principle that the target boundary is expressed as the envelope of the circles, with a center point $(X, 0)$ and radius $Z$. Fig. 7 shows the relationship between the target boundary and the envelopes of circles. Fig. 7 shows that the envelopes of the circles should circumscribe or inscribe to the target boundary, according to the sign of $\partial x / \partial X=1-Z \partial^{2} Z / \partial X^{2}-(\partial Z / \partial X)^{2}$, which denotes the target curvature. This method also requires the quasi-wavefront to create the envelope of circles. This method approximates a target region $(x, z)$ for each $(X, Z)$ as

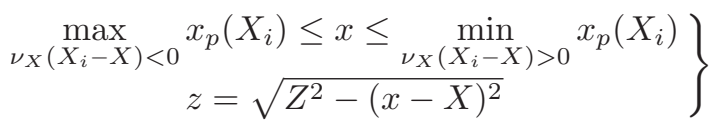

where $\nu_{X}=\operatorname{sgn}(\partial x / \partial X)$ and $X_{i}$ is a searching variable. $x_{p}\left(X_{i}\right)$ is defined as the intersection point between the circles, determined with $(X, Z)$ and $\left(X_{i}, Z_{i}\right)$, as shown in Fig. 7. Equation (7) enables group mapping from the points $(X, Z)$ to the points $(x, z)$ without derivatives. Thus, the instability caused by range fluctuation is suppressed.

It is confirmed that this method achieves high-speed and stable imaging for a simple target boundary. However, as shown in Fig. 8, the image produced by Envelope is inaccurate for the complex one. This is because Envelope requires appropriately connected range points to obtain an accurate image. Complex targets, in general, have many scattering points on their surfaces, and each antenna observes many ranges. The connection procedure is a complicated problem because each point of $(X, Z)$ has multiple connecting candidates around

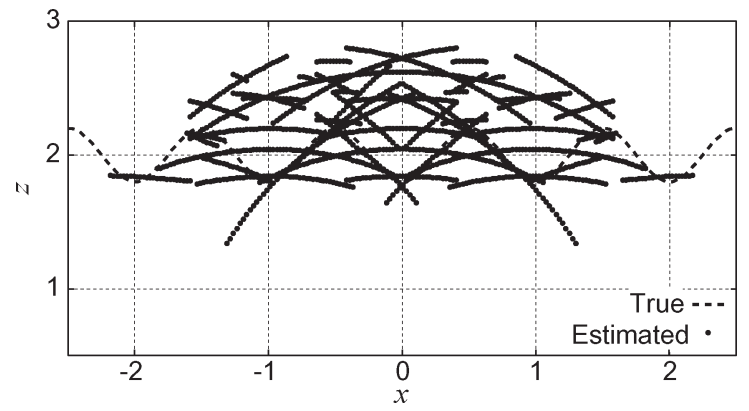

Fig. 8. Estimated image with Envelope.

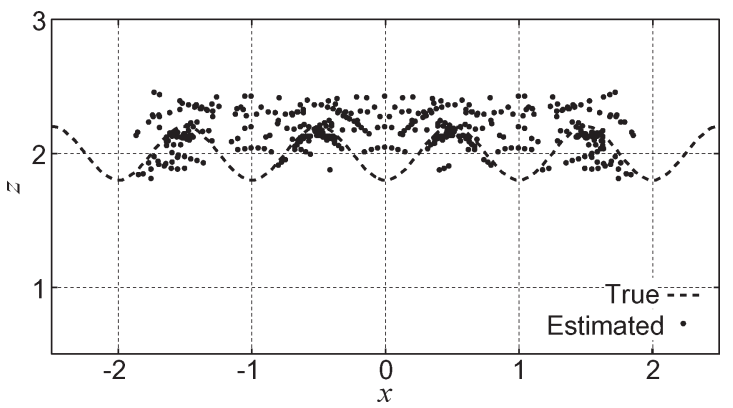

Fig. 9. Estimated image with Fourier transform and IBST.

itself. If the connection fails, the image estimated by Envelope has nonnegligible errors because it uses an incorrect envelope of circles with mistakingly determined $\nu_{X}$.

4) IBST With Fourier Transform: An imaging algorithm using Fourier transform and IBST realizes stable imaging because it does not require range point connections. The range inclination $\partial Z / \partial X$ can be calculated with the received 2-D data $s\left(X, Z^{\prime}\right)$. In this method, $\partial Z / \partial X$ for each point $(X, Z)$ is approximated as

$$
\partial Z / \partial X \simeq-\hat{k_{X}} / \hat{k_{Z}}
$$

where $\hat{k_{X}}$ and $\hat{k_{Z}}$ are calculated as

$$
\left(\hat{k_{X}}, \hat{k_{Z}}\right)=\arg \max _{k_{X}, k_{Z}}\left|S\left(k_{X}, k_{Z}\right)_{(X, Z)}\right| .
$$

Here, $S\left(k_{X}, k_{Z}\right)_{(X, Z)}$ is the 2-D Fourier transform of the spatially filtered signal around $(X, Z)$ given as

$$
\begin{array}{r}
S\left(k_{X}, k_{Z}\right)_{(X, Z)}=\int_{-\infty}^{\infty} \int_{-\infty}^{\infty} \Gamma\left(X^{\prime}, Z^{\prime} ; X, Z\right) s\left(X^{\prime}, Z^{\prime}\right) \\
\cdot e^{-j\left(k_{X} X^{\prime}+k_{Z} Z^{\prime}\right)} d X^{\prime} d Z^{\prime}
\end{array}
$$

where the gate function $\Gamma\left(X^{\prime}, Z^{\prime} ; X, Z\right)$ is defined as

$$
\Gamma\left(X^{\prime}, Z^{\prime} ; X, Z\right)=\exp \left\{-\frac{\left(X-X^{\prime}\right)^{2}+\left(Z-Z^{\prime}\right)^{2}}{2 \sigma_{F}^{2}}\right\} .
$$

The derivative for each range point can be stably calculated using the pulse waveform and amplitude. Fig. 9 shows the image estimated by combining the 2-D Fourier transform and IBST for the same data in Fig. 3. $\sigma_{F}=0.2 \lambda$ is set. The figure 


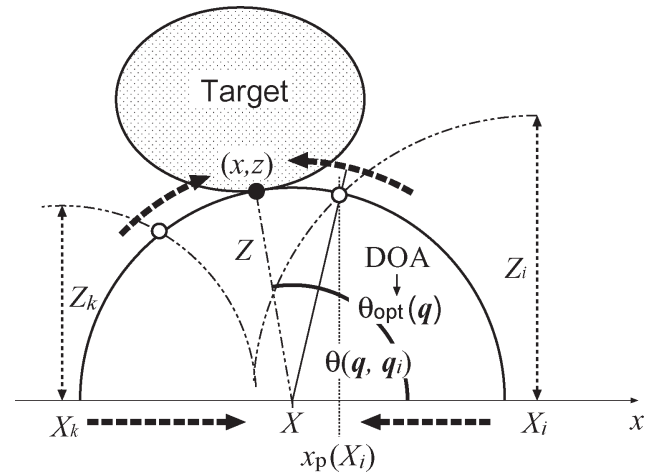

Fig. 10. Relationship between the target boundary and the convergence orbit of the intersection points.

shows that the obtained image has many inaccurate points and that the correct target boundary is hardly reconstructed. This is because the accuracy for $\partial Z / \partial X$ is affected by waveform deformations caused by interferences from multiple scatterers. Moreover, the method requires $240 \mathrm{~s}$ for imaging, which is not suitable for the real-time application.

\section{Proposed Algorithm}

1) Principle of the Proposed Algorithm: This section describes a proposed imaging algorithm to resolve the problems of the conventional algorithms. The proposed algorithm is based on the simple principle that a target boundary point should exist on a circle, with a center at $(X, 0)$ and radius of $Z$. Thus, each point $(x, z)$ can be calculated using the corresponding angle of arrival, defined as DOA. For the DOA estimation, the following function $f\left(\theta, \boldsymbol{q}, \boldsymbol{q}_{i}\right)$ is introduced:

$f\left(\theta, \boldsymbol{q}, \boldsymbol{q}_{i}\right)=\exp \left[-\frac{\left\{\theta-\theta\left(\boldsymbol{q}, \boldsymbol{q}_{i}\right)\right\}^{2}}{2 \sigma_{\theta}^{2}}\right], \quad\left(X \neq X_{i}\right)$

where $\boldsymbol{q}=(X, Z), \boldsymbol{q}_{i}=\left(X_{i}, Z_{i}\right)$, and $\theta\left(\boldsymbol{q}, \boldsymbol{q}_{i}\right)$ denotes the angle from the $x$-axis to the intersection point of the circles, with parameters $(X, Z)$ and $\left(X_{i}, Z_{i}\right)$. Any function with a center peak and symmetric curve, such as the raised cosine function, can be used in (12) instead of the Gaussian function. Fig. 10 shows the relationship between the intersection points of the circles and the angle of arrival.

Let us now introduce the following proposition in order to verify the suitability of the evaluation function $F(\theta ; \boldsymbol{q})$, which is subsequently defined. We assume that $\left(X_{i}, Z_{i}\right)$ moves to $(X, Z)$ along an exact quasi-wavefront, as defined in [16]. Under this condition, the following proposition holds.

Proposition 1: If $\left|X-X_{i}\right| \leq\left|X-X_{j}\right|$ and $\left(X-X_{i}\right)(X-$ $\left.X_{j}\right) \geq 0$ are satisfied, then

$$
\left|x-x_{p}\left(X_{i}\right)\right| \leq\left|x-x_{p}\left(X_{j}\right)\right|
$$

where $x=X-Z \partial Z / \partial X$. Proposition 1 is proved in the Appendix. This proposition states that, as $X_{i}$ moves to $X$, the distance between $x$ of the target point and $x_{p}\left(X_{i}\right)$ decreases. $\theta\left(\boldsymbol{q}, \boldsymbol{q}_{i}\right)$ then converges to the true angle of arrival. According
TABLE I

Required Procedures IN EACH Method

\begin{tabular}{|l|c|c|c|}
\hline & SEABED & Envelope & Proposed \\
\hline Derivative Operation & Required & Not Required & Not Required \\
\hline Range Points Connection & Required & Required & Not Required \\
\hline
\end{tabular}

to these conditions, the evaluation value $F(\theta ; \boldsymbol{q})$ for the angle estimation is introduced as

$$
F(\theta ; \boldsymbol{q})=\left|\sum_{i=1}^{N_{q}} s\left(X_{i}, Z_{i}\right) f\left(\theta, \boldsymbol{q}, \boldsymbol{q}_{i}\right) e^{-\frac{\left(X-X_{i}\right)^{2}}{2 \sigma_{X}^{2}}}\right|
$$

where the constants $\sigma_{\theta}$ and $\sigma_{X}$ are empirically determined and $N_{q}$ is the total number of the range points. The weight function $\exp \left\{-\left(X-X_{i}\right)^{2} / 2 \sigma_{X}^{2}\right\}$ in (14) yields a convergence effect of intersection points to the angle estimation given in Proposition 1. The optimum DOA for each $\boldsymbol{q}$ is calculated as

$$
\theta_{\mathrm{opt}}(\boldsymbol{q})=\arg \max _{\theta} F(\theta ; \boldsymbol{q})
$$

The target boundary $(x, z)$ for each range point $(X, Z)$ is expressed as $x=X+Z \cos \theta_{\text {opt }}(\boldsymbol{q})$ and $z=Z \sin \theta_{\text {opt }}(\boldsymbol{q})$. A noteworthy advantage of this algorithm is that the optimum DOA can be calculated without connecting the range points in (14). This method realizes direct mapping from the points of range to the points of target boundary without range point connections or derivative operations. Thus, the inaccuracy occurring in the conventional algorithms can be substantially avoided with this method.

2) Procedure of the Proposed Method:

Step 1) Calculate $F(\theta ; \boldsymbol{q})$ in (14) for $\boldsymbol{q}=(X, Z)$.

Step 2) Obtain $\theta_{\text {opt }}(\boldsymbol{q})$ in (15), where $\zeta(\boldsymbol{q})$ is set as

$$
\zeta(\boldsymbol{q})=F\left(\theta_{\mathrm{opt}}(\boldsymbol{q}) ; \boldsymbol{q}\right) .
$$

Step 3) Calculate the point on the target boundary $(x, z)$ as

$$
\left.\begin{array}{ccc}
x & = & X+Z \cos \theta_{\mathrm{opt}}(\boldsymbol{q}) \\
z & = & Z \sin \theta_{\mathrm{opt}}(\boldsymbol{q})
\end{array}\right\} .
$$

Step 4) Carry out steps 1)-3) for all range points, and obtain target points.

Step 5) Remove the target points that satisfy

$$
\zeta(\boldsymbol{q}) \leq \beta \max _{i} \zeta\left(\boldsymbol{q}_{i}\right) .
$$

$\beta$ is empirically determined.

Step 5) Suppresses false images caused by random noise, where its $\zeta(\boldsymbol{q})$ becomes smaller than that of a true image in most case. In this procedure, the proposed method determines each target point $(x, z)$ by only finding $\theta$ which maximizes the evaluation function $F(\theta ; \boldsymbol{q})$, and then, it can avoid the difficulty of connecting range points.

Table I shows the required procedures for each method. The processes "Derivative Operation" and "Range Point Connection" yield inaccuracy and difficulties for imaging in the case of complex boundaries, as described in Section II-B. The proposed method does not require these procedures, and inaccuracy can be substantially resolved. 


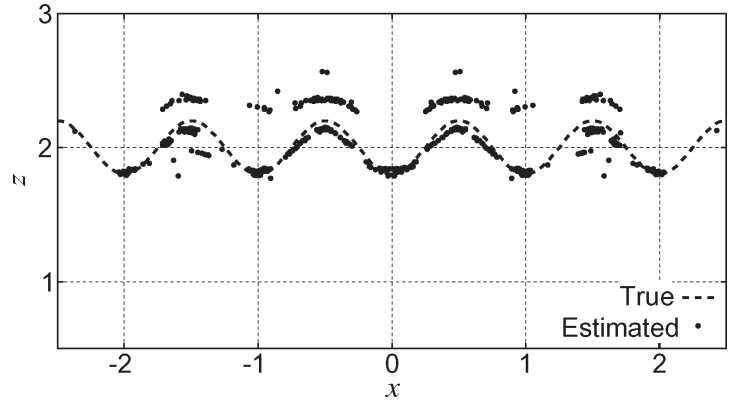

Fig. 11. Estimated image with the proposed method.

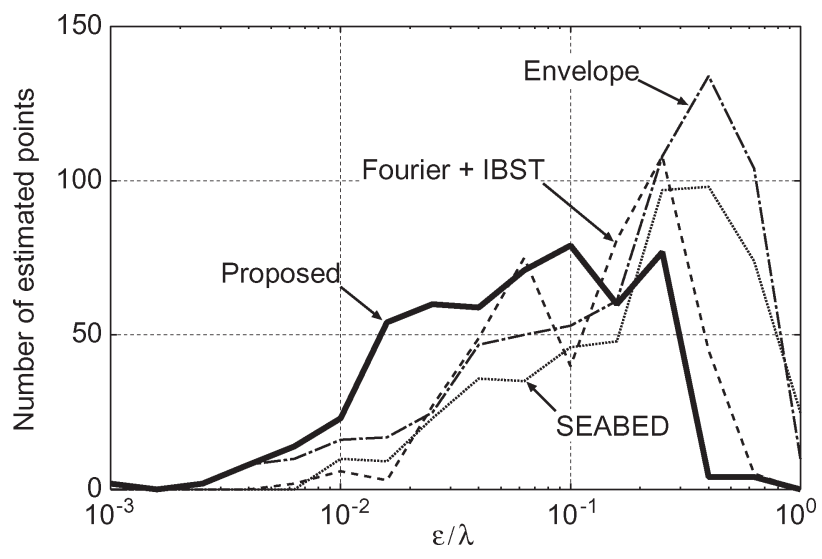

Fig. 12. Error distribution for each method at the complex target.

\section{Performance Evaluation of the Numerical Simulation}

1) Complex Boundary: Fig. 11 shows the image estimated by the proposed method for the same data as those in Fig. 3. $\sigma_{X}=1.0 \lambda, \sigma_{\theta}=\pi / 50, \alpha=0.2$, and $\beta=0.3$ are set. A discussion of the parameter selection is given in the latter part of this section. This result verifies that the proposed method significantly enhances the accuracy of the estimated points, even for a complex target boundary. It also shows that there are false images above the target boundary caused by the multiple scattered signals, which we aim to suppress in the future. For a quantitative evaluation of the accuracy, $\epsilon\left(\boldsymbol{x}_{e}^{i}\right)$ is introduced as

$$
\epsilon\left(\boldsymbol{x}_{e}^{i}\right)=\min _{\boldsymbol{x}}\left\|\boldsymbol{x}-\boldsymbol{x}_{e}^{i}\right\|^{2}, \quad\left(i=1,2, \ldots, N_{T}\right) .
$$

Here, $\boldsymbol{x}$ and $\boldsymbol{x}_{e}^{i}$ express the location of the true target point and that of the estimated point, respectively. $N_{T}$ is the total number of $\boldsymbol{x}_{e}^{i}$. Fig. 12 shows the number of the estimated points for each value of $\epsilon$. SAR is discarded in this evaluation to allow the comparison of only those methods using range points $(X, Z)$. The value of $N_{T}$ for each algorithm is 521 for SEABED, 430 for Envelope, 375 for IBST+Fourier, and 517 for the proposed method. The figure reveals that the number of estimated points with $\epsilon \leq 0.1 \lambda$ is significantly larger than the numbers for the other algorithms. Furthermore, the detection probability $P_{D}$ of each algorithm is investigated, where $P_{D}$ is defined as the ratio of the number of the estimated points with $\epsilon \leq 0.1 \lambda$ to $N_{T}$. The $P_{D}$ values for the algorithms are 0.316 for SEABED, 0.353 for Envelope, 0.470 for IBST+Fourier, and 0.720 for the proposed method. This result also verifies that the proposed algorithm enhances the accuracy remarkably in terms of its ability to

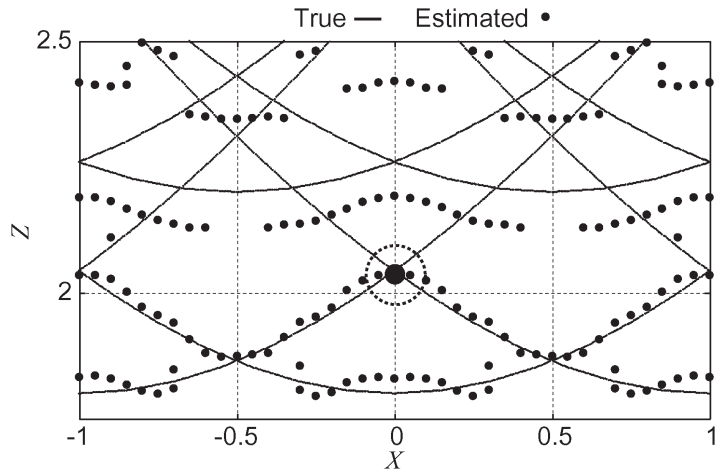

Fig. 13. Enlarged view for the range points in Fig. 3.

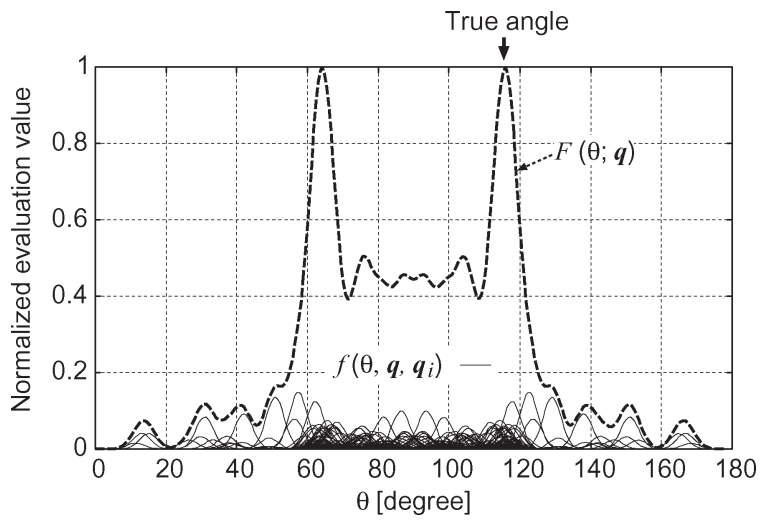

Fig. 14. Evaluation example for $f\left(\theta, \boldsymbol{q}, \boldsymbol{q}_{i}\right)$ and $F(\theta ; \boldsymbol{q})$ at $\boldsymbol{q}=(0.0,2.05)$.

detect targets. It should, however, be noted that this algorithm does not improve the spatial resolution, which is strictly limited to one-half of the wavelength of the transmitted pulse. However, the proposed method can provide more significant images to recognize target shapes with clear surfaces, by increasing the number of accurate boundary points.

Here, we discuss the effectiveness of the proposed method. Fig. 13 shows an enlarged illustration of the range points in Fig. 3. In this figure, two of the true quasi-wavefronts (solid lines) intersect at point $\boldsymbol{q}=(0.0,2.05)$. Then, the derivatives $\partial Z / \partial X$ for the two wavefronts are around 0.4 and -0.4 , respectively, which correspond to approximately $65^{\circ}$ or $115^{\circ}$ using the relationship $\theta=\cos ^{-1}(-\partial Z / \partial X)$ [16]. However, the observed range points (black dots) around $\boldsymbol{q}=(0.0,2.05)$ are not on the true quasi-wavefronts, because multiple scattered waves are included in ranges that are smaller than the wavelength and the peak estimations have nonnegligible errors. Then, the estimated derivative $\partial Z / \partial X$ is around zero, which corresponds to an incorrect DOA of $90^{\circ}$. Contrarily, Fig. 14 shows each value of $f\left(\theta, \boldsymbol{q}, \boldsymbol{q}_{i}\right)$ and $F(\theta ; \boldsymbol{q})$ for $\boldsymbol{q}=(0.0,2.05)$, and we recognize the maximum for $\theta=65^{\circ}$ or $\theta \simeq 115^{\circ}$ around the true angle using (15) in the proposed method. This is because each angle of arrival can be calculated from the global distribution of the range points, and this avoids inaccuracy in the angle of arrival due to the derivative or misconnection of the range points.

The sensitivity of the proposed method with respect to parameters $\alpha, \beta, \sigma_{X}$, and $\sigma_{\theta}$ is investigated as follows. The graphs on the left- and right-hand sides of Fig. 15 show $P_{D}$ 

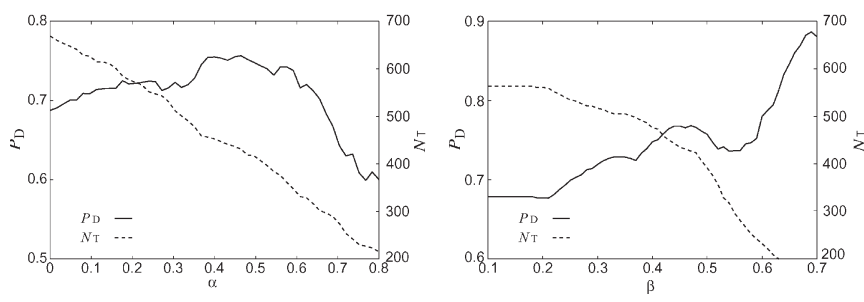

Fig. 15. Sensitivity evaluations of the proposed method for (left) $\alpha$ and (right) $\beta$.
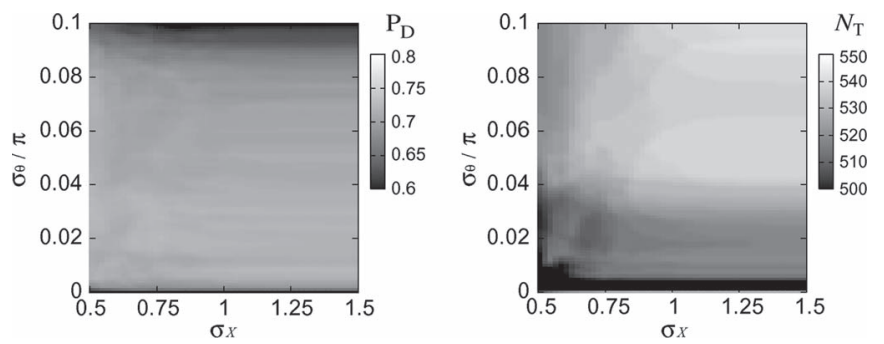

Fig. 16. Sensitivity evaluation of the proposed method for $\sigma_{X}$ and $\sigma_{\theta}$ in (left) $P_{D}$ and (right) $N_{T}$.

and $N_{T}$ values for $\alpha$ and $\beta$, respectively, given that $\sigma_{\theta}=\pi / 50$ and $\sigma_{X}=1.0 \lambda$. In both graphs, $N_{T}$ decreases as either $\alpha$ or $\beta$ increases. This is because $\alpha$ limits the number of range points in the peak extraction of $s\left(X, Z^{\prime}\right)$ in (3). Moreover, $\beta$ regulates the threshold of selecting the estimated points in (18). On the other hand, Fig. 15 shows that $P_{D}$ increases gently for $\alpha \leq 0.6$, because a large $\alpha$ suppresses the extraction of false ranges from unnecessary responses of the Wiener filter in Fig. 3. It also shows that $P_{D}$ increases as $\beta$ increases, because more accurate points have a higher value of $F(\theta ; \boldsymbol{q})$. Considering the balance between the $P_{D}$ and $N_{T}$ values, $\alpha$ and $\beta$ should be set to about 0.2 and 0.3 , respectively. The left- and righthand sides of Fig. 16 show the $P_{D}$ and $N_{T}$ values for each $\sigma_{X}$ and $\sigma_{\theta}$, given that $\alpha=0.2$ and $\beta=0.3$. The left-hand side of Fig. 16 shows that, although $P_{D}$ decreases as $\sigma_{\theta}$ increases, $P_{D}$ does not depend on $\sigma_{X}$. This is because the larger $\sigma_{\theta}$ blurs the peak of (14) and distorts the accuracy for $\theta_{\mathrm{opt}}$. On the other hand, the right-hand side of Fig. 16 shows that $N_{T}$ has an increasing tendency for the both $\sigma_{\theta}$ and $\sigma_{X}$. From these results, the appropriate parameters have been determined as $\sigma_{\theta}=\pi / 50$ and $\sigma_{X}=1.0 \lambda$. Furthermore, by using these same parameters, we have confirmed that the proposed algorithm produces accurate images for various kinds of target shapes with edge, plate, concave, or convex boundaries. Thus, the selection of parameter values is not substantially affected by the target shapes.

It is shown that the calculation time of the proposed method is within $0.2 \mathrm{~s}$ for processing on a Xeon $2.8-\mathrm{GHz}$ computer, which is suitable for real-time operation. In addition, Table II shows the comparison of the calculation time and computational complexity for each algorithm, where $N_{X}, N_{Z^{\prime}}, N_{x}$, and $N_{z}$ denote the sampling numbers of $X, Z^{\prime}, x$, and $z$, respectively, and $O(*)$ gives the Landau notation. The highspeed imaging is possible because the method uses only the ranges and amplitudes, and this decreases the calculation cost for the boundary extraction when compared with SAR and other data synthesis algorithms.
TABLE II

Calculation Time (For XeOn 2.8-GHz Processor) AND COMPUTER COMPLEXITY OF EACH Algorithm

\begin{tabular}{|l|c|c|}
\hline & Calculation time & Computational complexity \\
\hline SAR & $60 \mathrm{sec}$ & $O\left(N_{X} N_{Z^{\prime}} N_{x} N_{z}\right)$ \\
\hline FK-migration & $2.0 \mathrm{sec}$ & $O\left(N_{X} N_{Z^{\prime}} \log N_{X} N_{Z^{\prime}}\right)$ \\
\hline SEABED & $0.1 \mathrm{sec}$ & $O\left(N_{\mathrm{q}}\right)$ \\
\hline Envelope & $0.2 \mathrm{sec}$ & $O\left(N_{\mathrm{q}}\right)$ \\
\hline IBST+Fourier & $240 \mathrm{sec}$ & $O\left(N_{\mathrm{q}} N_{X} N_{Z^{\prime}} \log N_{X} N_{Z^{\prime}}\right)$ \\
\hline Proposed method & $0.2 \mathrm{sec}$ & $O\left(N_{\mathrm{q}}^{2}\right)$ \\
\hline
\end{tabular}

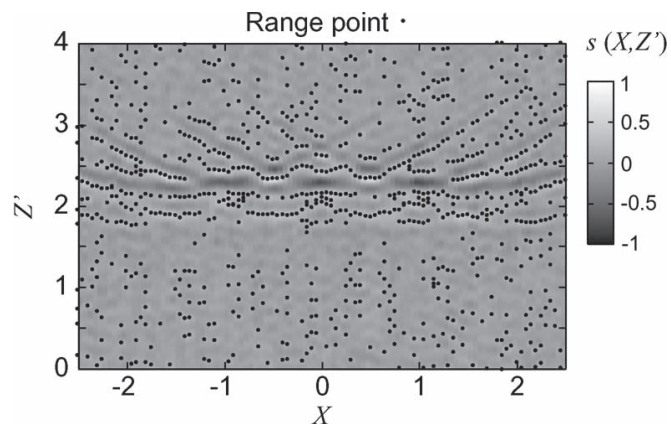

Fig. 17. Output of the Wiener filter and the extracted range points for $S / N=$ $20 \mathrm{~dB}$.

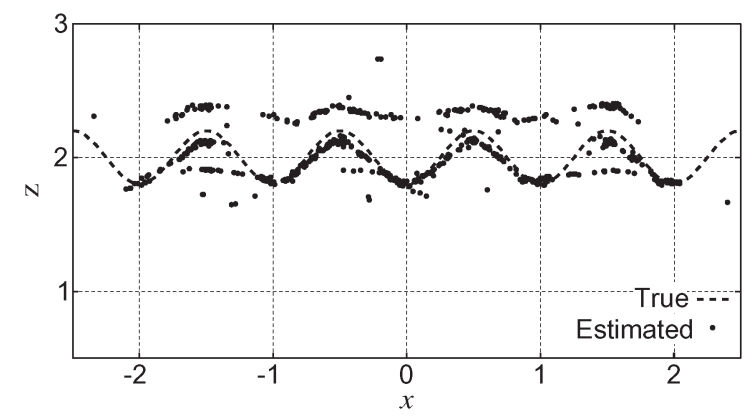

Fig. 18. Estimated image with the proposed method for $S / N=20 \mathrm{~dB}$

2) Performance in Noisy Situation: Next, the application example of a noisy situation is presented. Fig. 17 shows the output of the Wiener filter and the extracted range points for $S / N=20 \mathrm{~dB}$. Here, $S / N$ is defined as the ratio of the peak instantaneous signal power to the averaged noise power after applying the matched filter. As shown in Fig. 17, there are many incorrect range points due to the random noise. Fig. 18 shows the target image obtained with the proposed method. Although there are some false images due to random noise, for example $(x, z)=(-0.25,2.5)$, an accurate image is still produced even in a noisy environment. The accuracy for each method is quantitatively compared for various noisy cases using the evaluation value $\mu$, which is defined as the root mean squares of $\epsilon\left(\boldsymbol{x}_{e}^{i}\right)$ defined in (19). Fig. 19 shows the relationship between $S / N$ and $\mu$ for each method. The figure verifies that the proposed method provides an accurate image in noisy situations, and $\mu$ holds within $0.15 \lambda$ for $S / N \geq 20 \mathrm{~dB}$. Here, it should be noted that the assumed target boundary represents one of the most difficult cases with the surface variation around $0.4 \lambda$, because multiple interferences from the different scattering points on this target surface are included in the received signal for ranges that are smaller than wavelength. These interferences create a complicated distribution of $(X, Z)$ as in Fig. 3, because 


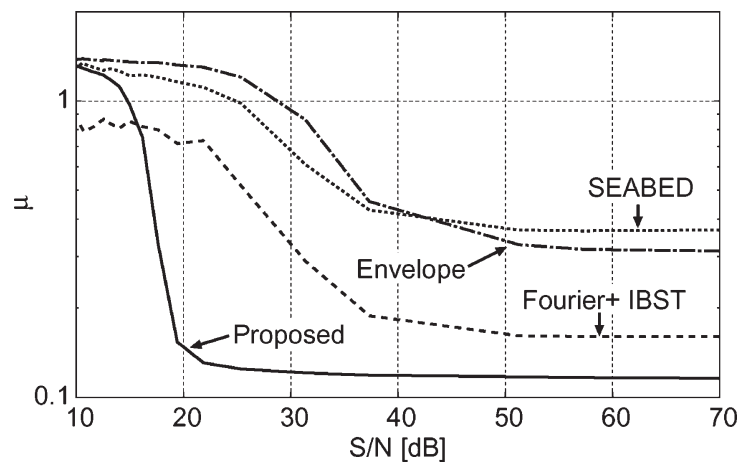

Fig. 19. $\mu$ for each $S / N$ at the complex target boundary.

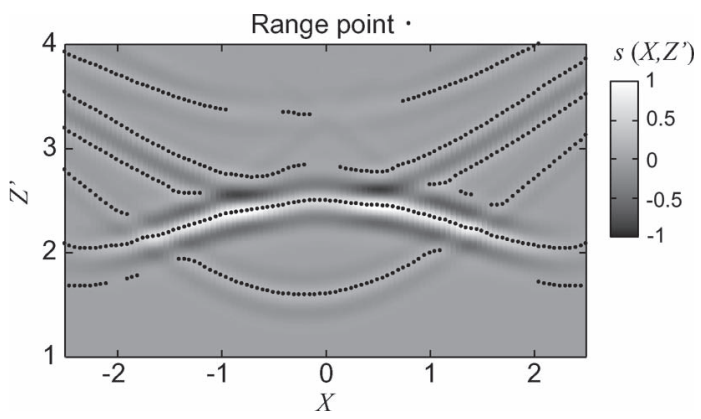

Fig. 20. Output of the Wiener filter and the extracted range points for the small circle and concave boundary.

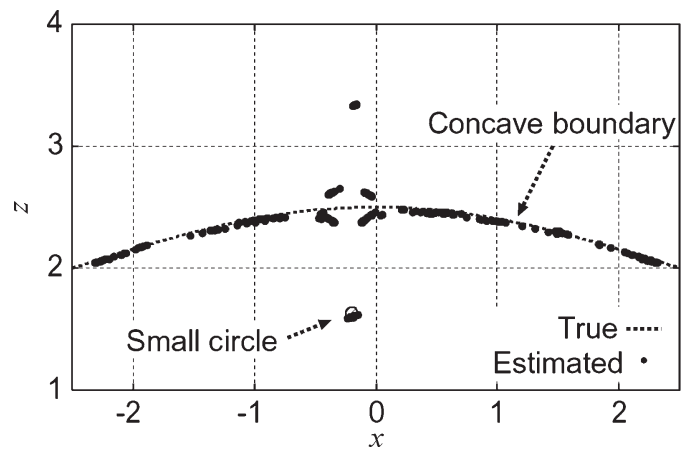

Fig. 21. Estimated image with the proposed method for the small circle and concave boundary.

multiple range points converge in the small region of $(X, Z)$. This distribution, therefore, causes serious errors in images obtained using the conventional algorithms as shown in Figs. 6, 8 , and 9. On the contrary, the proposed method improves the accuracy remarkably compared to the conventional algorithms, even with one of the most difficult target shapes.

3) Multiple Boundaries: We show that the proposed algorithm achieves accurate imaging, where multiple boundaries with large and small curvatures are intermingled. Fig. 20 shows the output of the Wiener filter in the case of the concave boundary and small circle. Fig. 21 shows the produced image by the proposed method. The figure confirms that the image accurately expresses both target boundaries. This is because the evaluation value $F(\theta ; \boldsymbol{q})$ in (14) depends on the received amplitude $s(X, Z)$ and the density of the intersection points between the circles. Although the power of each received signal from the small circle is smaller than that from the concave

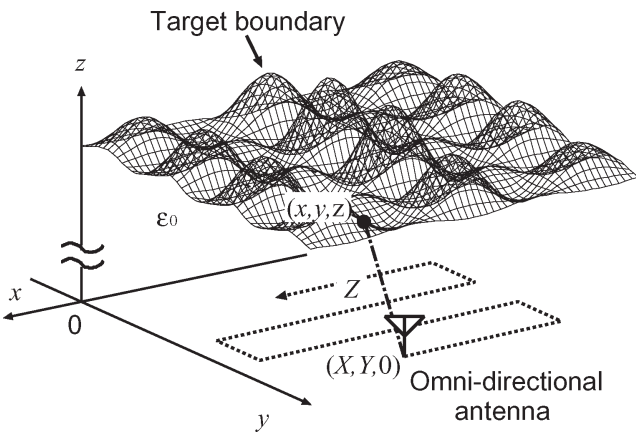

Fig. 22. System model in 3-D problem.

boundary, the density of the intersection points of the small circle is greater than that of the concave boundary above. Thus, in the case of the small circle or the concave boundary, $F(\theta ; \boldsymbol{q})$ creates a significant peak for each DOA.

\section{3-D PROBLEM}

\section{A. System Model}

Fig. 22 shows the system model for a 3-D problem. The target model, antenna, and transmitted signal are the same as those assumed in the 2-D problem. The antenna is scanned along the plane, $z=0$. We assume a linear polarization in the direction of the $x$-axis. The $\mathrm{r}$-space is expressed by the parameter $(x, y, z)$. We assume $z>0$ for simplicity. $s^{\prime}(X, Y, t)$ is defined as the received electric field at the antenna location $(x, y, z)=(X, Y, 0)$. The output of the Wiener filter $s\left(X, Y, Z^{\prime}\right)$ is obtained by the same procedure described in Section II-A. We connect the significant peaks of $s\left(X, Y, Z^{\prime}\right)$ as $Z$ for each $X$ and $Y$ and extract range point as $(X, Y, Z)$ by using the same criteria in (3). The d-space is defined as the space expressed by $(X, Y, Z)$.

\section{B. Conventional Algorithms}

1) SEABED: The SEABED algorithm for 3-D problems has been developed. It achieves real-time and nonparametric 3-D imaging with IBST [14]. The IBST from the quasi-wavefront $(X, Y, Z)$ to the target boundary $(x, y, z)$ is formulated as

$$
\left.\begin{array}{l}
x=X-Z \partial Z / \partial X \\
y=Y-Z \partial Z / \partial Y \\
z=Z \sqrt{1-(\partial Z / \partial X)^{2}-(\partial Z / \partial Y)^{2}}
\end{array}\right\} .
$$

This transform gives us a direct solution for the clear boundary extraction. An application example of SEABED is presented as follows. We assume a complex target boundary with sinusoidal surfaces as shown in Fig. 22, which is defined as $z=1.2-0.3\{\cos (\pi x)+0.5\}\{\cos (1.5 \pi y)+$ $0.5\} e^{-\left(x^{2}+y^{2}\right) / 9}$. The range points are extracted from the received data calculated by the FDTD method, and the quasiwavefront is created by connecting these points. Figs. 23 and 24 show the outputs of the Wiener filter and the extracted range points $(X, Y, Z)$ at $X=0$ and $Y=0.6$, respectively. We take the received signal at 51 locations for $-2.5 \leq x, y \leq 2.5$. Fig. 25 shows the estimated images at $x=0$ and $y=0.6$, 


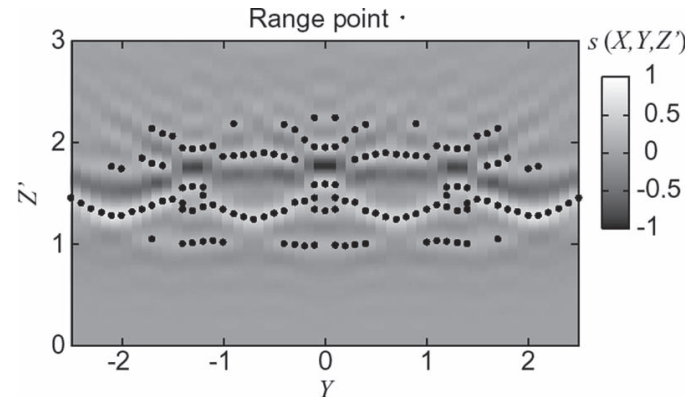

Fig. 23. Output of the Wiener filter and extracted range points at $X=0$.

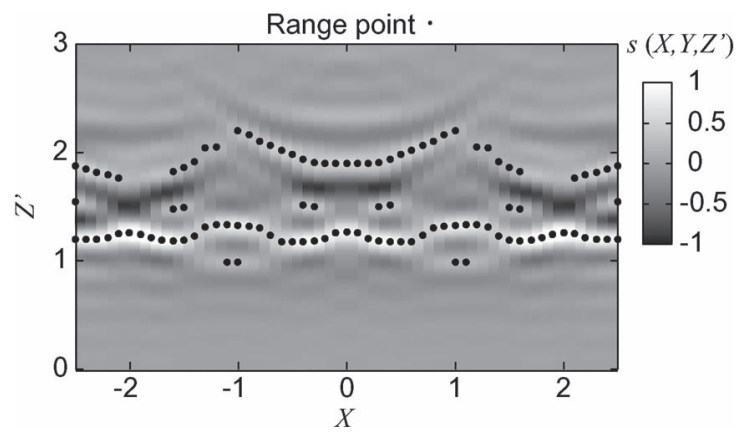

Fig. 24. Output of the Wiener filter and extracted range points at $Y=0.6$.
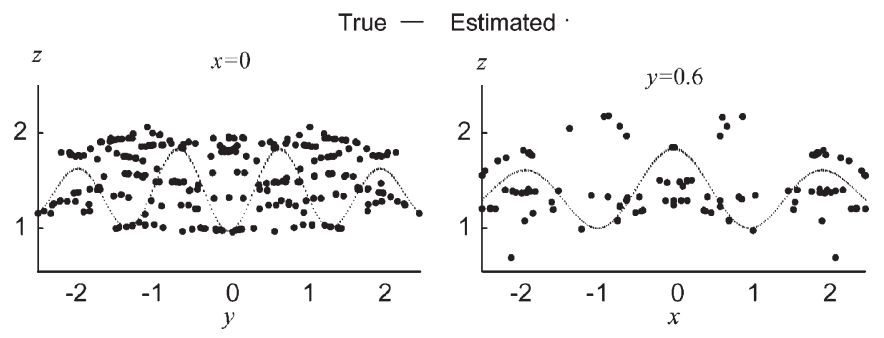

Fig. 25. Estimated image with SEABED for the 3-D complex target.

respectively, using SEABED. The figure shows that there is a nonnegligible fluctuation of the estimated points because the image estimated by SEABED strongly depends on the accuracy of the derivative of $Z$, which is quite sensitive to small range errors due to interference.

2) Envelope: The Envelope method for 3-D problems has been proposed to realize stable and high-speed 3-D imaging with an envelope of spheres [17]. Similar to the 2-D problem, this method calculates the target boundary $(x, y, z)$ for each $(X, Y, Z)$ as

$$
\left.\begin{array}{c}
\max _{\nu_{X}\left(X_{i}-X\right)<0} x_{p}^{3 d}\left(X_{i}\right) \leq x \leq \min _{\nu_{X}\left(X_{i}-X\right)>0} x_{p}^{3 d}\left(X_{i}\right) \\
\max _{\nu_{Y}\left(Y_{i}-Y\right)<0} y_{p}^{3 d}\left(Y_{i}\right) \leq y \leq \min _{\nu_{Y}\left(Y_{i}-Y\right)>0} y_{p}^{3 d}\left(Y_{i}\right) \\
z=\sqrt{Z^{2}-(x-X)^{2}-(y-Y)^{2}}
\end{array}\right\}
$$

where $X_{i}$ and $Y_{i}$ are searching variables and $\nu_{Y}=$ $\operatorname{sgn}(\partial y / \partial Y) \cdot y_{p}^{3 d}\left(Y_{i}\right)$ is defined as the intersection point between the projected circles of two spheres determined by $(X, Y, Z)$ and $\left(X, Y_{i}, Z_{i}\right)$ on the plane $x=X . x_{p}^{3 d}\left(X_{i}\right)$ is defined similarly on the plane $y=Y$. Equation (21) determines an arbitrary target boundary without derivative operations, which can suppress the instability caused by small range errors.

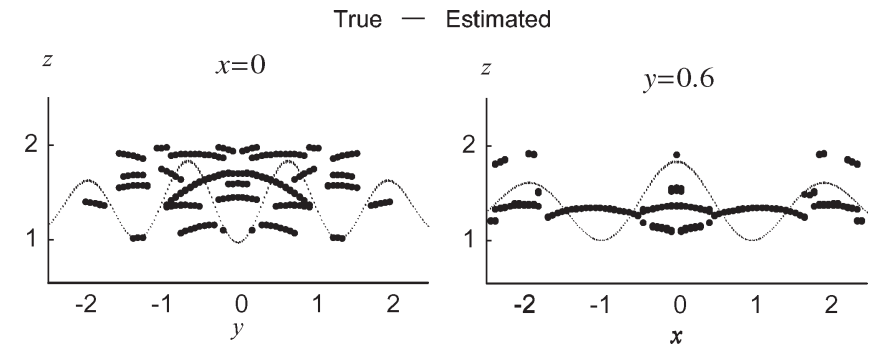

Fig. 26. Estimated image with Envelope for the 3-D complex target.

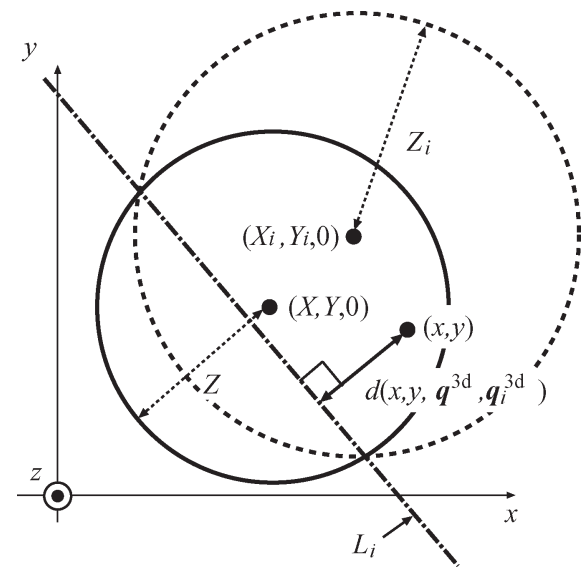

Fig. 27. Intersection line $L_{i}$ of two spheres on $z=0$ plane.

Fig. 26 shows the image obtained with Envelope from the same views as those in Fig. 25. It is confirmed that the fluctuation of the estimated points is suppressed without the use of the range derivative. However, there are significant image distortions because the misconnection of the range points produces an incorrect envelope of spheres, which results in the large errors for the estimated regions. This determination requires a correct wavefront connection for the range points, and it often becomes more difficult than that in a 2-D problem because each point must be correctly connected along both the $x$ - and $y$-axes.

\section{Proposed Imaging Algorithm}

To resolve the previous problems, we extend the proposed method to 3-D modeling. In this model, the orbit of intersection points between two spheres for $(X, Y, Z)$ and $\left(X_{i}, Y_{i}, Z_{i}\right)$ becomes a circle. The projected curve of this circle on $z=0$ becomes a straight line. We define this line as $L_{i}$. Fig. 27 shows the intersection line $L_{i}$ of two spheres on the $z=0$ plane. Here, each angle of arrival corresponds to the location for $(x, y)$ for the assumption $z \geq 0$. This method determines the target location $(x, y)$ by using the evaluation function, defined as

$$
f\left(x, y, \boldsymbol{q}^{3 d}, \boldsymbol{q}_{i}^{3 d}\right)=\exp \left\{-\frac{d\left(x, y, \boldsymbol{q}^{3 d}, q_{i}^{3 d}\right)^{2}}{2 \sigma_{d}^{2}}\right\}
$$

where $\boldsymbol{q}^{3 d}=(X, Y, Z), \boldsymbol{q}_{i}^{3 d}=\left(X_{i}, Y_{i}, Z_{i}\right)$, and $d\left(x, y, \boldsymbol{q}^{3 d}\right.$, $\left.\boldsymbol{q}_{i}^{3 d}\right)$ denotes the minimum distance between the projected line $L_{i}$ and $(x, y)$. We use the extended principle that if $\left(X_{i}, Y_{i}, Z_{i}\right)$ moves to $(X, Y, Z)$ along an exact quasi-wavefront, $(x, y)$ 

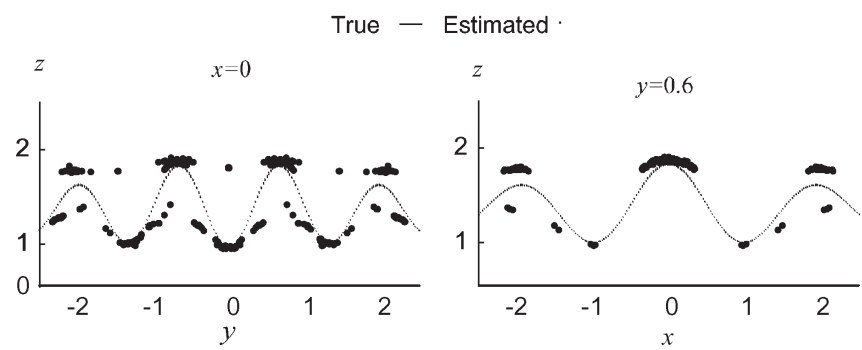

Fig. 28. Estimated image with the proposed method for the 3-D complex target.

converges to that of the true target point. For accurate locating of $(x, y)$, the evaluation value $F^{3 d}\left(x, y ; \boldsymbol{q}^{3 d}\right)$ is introduced as

$$
\begin{aligned}
& F^{3 d}\left(x, y ; \boldsymbol{q}^{3 d}\right) \\
& \quad=\left|\sum_{i=1}^{N q} s\left(X_{i}, Y_{i}, Z_{i}\right) f\left(x, y, \boldsymbol{q}^{3 d}, \boldsymbol{q}_{i}^{3 d}\right) e^{-\frac{D\left(\boldsymbol{q}^{3 d}, \boldsymbol{q}_{i}^{3 d}\right)^{2}}{2 \sigma_{D}^{2}}}\right|
\end{aligned}
$$

where $D\left(\boldsymbol{q}^{3 d}, \boldsymbol{q}_{i}^{3 d}\right)=\sqrt{\left(X-X_{i}^{2}\right)+\left(Y-Y_{i}^{2}\right)}, \sigma_{d}$, and $\sigma_{D}$ are empirically determined. The $x$ - and $y$-coordinates of the target boundary for each range point $\boldsymbol{q}^{3 d}$ are then calculated as

$$
\left(x\left(\boldsymbol{q}^{3 d}\right), y\left(\boldsymbol{q}^{3 d}\right)\right)=\arg \max _{x, y} F^{3 d}\left(x, y ; \boldsymbol{q}^{3 d}\right) .
$$

The $z$-coordinate of each target point is given by $z\left(\boldsymbol{q}^{3 d}\right)=$ $\sqrt{Z^{2}-\left\{x\left(\boldsymbol{q}^{3 d}\right)-X\right\}^{2}-\left\{y\left(\boldsymbol{q}^{3 d}\right)-Y\right\}^{2}}$. The method eliminates the connecting procedures of the range points, which can avoid inaccuracy due to the failure of range connections. Thus, it achieves a direct mapping from the all range points to the points of the target boundary without grouping.

\section{Performance Evaluation in Numerical Simulation}

This section presents an application example of a 3-D problem for the proposed method. Fig. 28 shows the image estimated by the proposed method. $\sigma_{d}=0.1 \lambda$ and $\sigma_{D}=0.5 \lambda$ are set. The method remarkably enhances the accuracy for 3-D complex target imaging. This is because it does not require range point connection and it eliminates inaccuracy due to an inappropriate range connection. Furthermore, the proposed method makes use of the distribution of the range points along not only the $x$ - and $y$-axes but also all directions to obtain an accurate target point. Fig. 29 shows the distribution of $\epsilon\left(\boldsymbol{x}_{e}^{i}\right)$ defined in (19) for the images estimated using each method. This figure quantitatively shows that the proposed method increases the number of the estimated points with $\epsilon \leq 0.1 \lambda$. The total number of the estimated points $N_{T}$ is 4928 for SEABED, 5626 for Envelope, and 5763 for the proposed algorithm. The detecting probability $P_{D}$ defined in Section II-D is 0.305 for SEABED, 0.468 for Envelope, and 0.544 for the proposed method. In addition, the proposed method obtains $\bar{\epsilon}=0.091 \lambda$, where $\bar{\epsilon}$ denotes the mean value of $\epsilon$. This value is superior to those in SEABED $(\bar{\epsilon}=0.215 \lambda)$ and Envelope $(\bar{\epsilon}=0.151 \lambda)$. The calculation time for this method is around $50 \mathrm{~s}$ for a Xeon $2.8-\mathrm{GHz}$ processor, because it requires a 2-D search for the assumed region for $(x, y)$ for each range point.

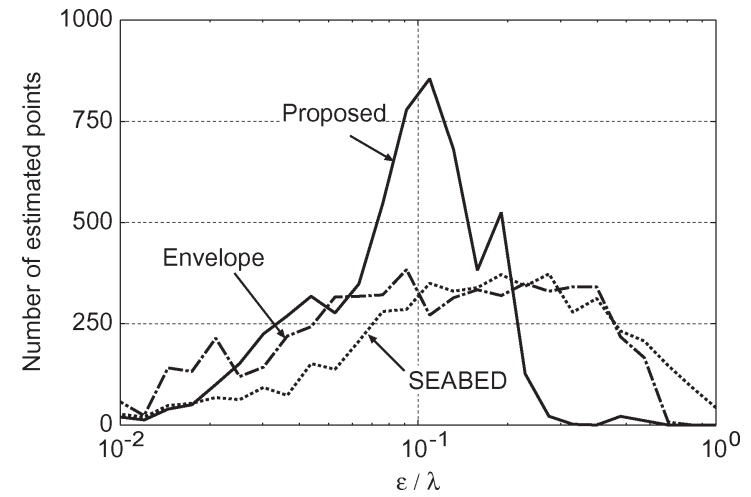

Fig. 29. Error distribution for each method at the 3-D complex target.

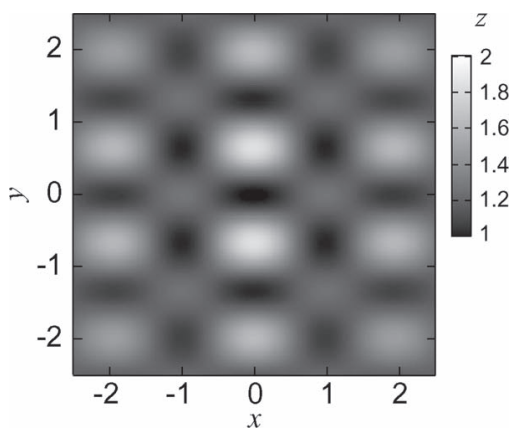

Fig. 30. True target contour image.

Next, the smoothing examples for the obtained images are presented to clearly show that our algorithm offers a higher quality 3-D image compared with the images for the conventional algorithms (Fig. 30). Here, we apply the simple smoothing algorithm by combining an extended median filter and the Gaussian function. First, we select the estimated points $\left(x_{i}, y_{i}, z_{i}\right)$, which are included in the preselected region for $(x, y)$, and $z_{i}$ is updated with other points included in this region as

$$
z_{i}^{\text {up }}=\left\{\begin{array}{ll}
z_{\text {med }}, & \left\{\begin{array}{l}
z_{i} \geq(1-\xi) \min _{j} z_{j}+\xi \max _{j} z_{j} \\
z_{i} \leq(1-\xi) \max _{j} z_{j}+\xi \min _{j} z_{j}
\end{array}\right. \\
z_{i}, & \text { (otherwise) }
\end{array}\right\}
$$

where $z_{\text {med }}$ is the median value for $z$ among the selected target points. $\xi$ is empirically determined. Second, the $z$-coordinate of the selected region $(x, y)$ is smoothed with the Gaussian function as

$$
z(x, y)=\frac{\sum_{i} z_{i}^{\text {up }} \exp \left\{-\frac{\left(x-x_{i}\right)^{2}+\left(y-y_{i}\right)^{2}}{2 \sigma_{I}^{2}}\right\}}{\sum_{i} \exp \left\{-\frac{\left(x-x_{i}\right)^{2}+\left(y-y_{i}\right)^{2}}{2 \sigma_{I}^{2}}\right\}} .
$$

Fig. 30 shows the true target boundary. The left- and righthand sides of Fig. 31 show the estimated contour image and its cross section along $x=0$ using SEABED, respectively. Fig. 32 shows the image estimated with Envelope from the same view as that in Fig. 31. $\sigma_{I}=0.1 \lambda$ and $\xi=0.2$ are set. The target surface is removed if the number of estimated points becomes zero in the selected region $(x, z)$. These figures show that the smoothed images with both methods hardly reconstruct a correct target boundary, and the characteristic 

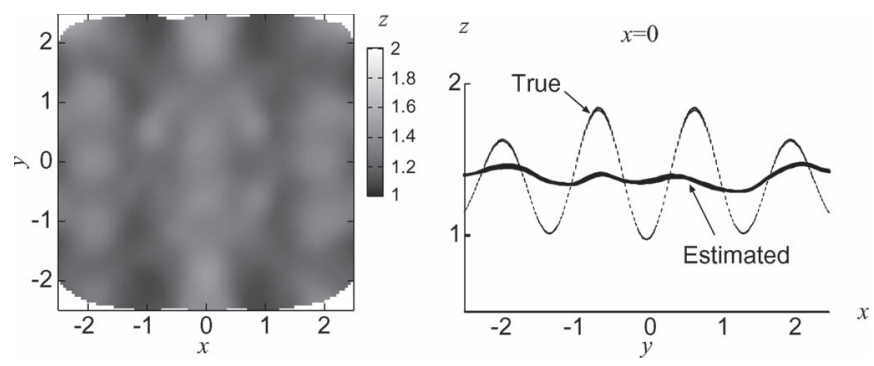

Fig. 31. (Left) Estimated contour image with SEABED after smoothing and (right) its cross-sectional view at $x=0$.
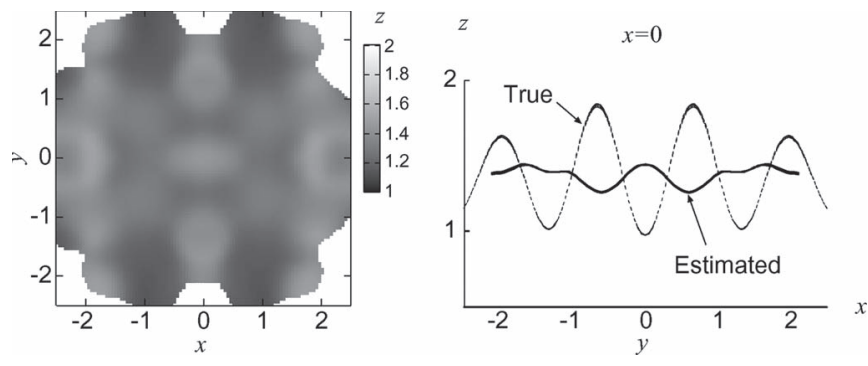

Fig. 32. Smoothed image with Envelope from the same view in Fig. 31.
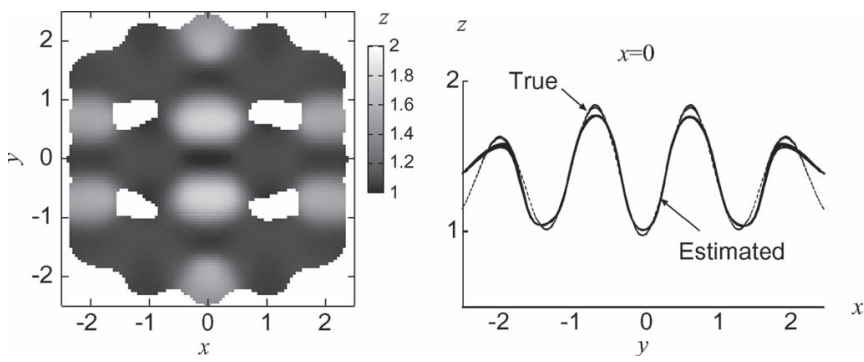

Fig. 33. Smoothed image with the proposed method from the same view in Fig. 31.

of the target boundary is lost by the smoothing of inaccurate points. Contrarily, Fig. 33 shows the image estimated by the proposed method from the same view as that in Figs. 31 and 32. The white symmetric patches in this figure show the removed surfaces in the smoothing process. The figure confirms that the image smoothing is effective for the proposed method, and the target boundary can be accurately reconstructed even for complex targets. This result shows that there is a remarkable advantage in using the proposed method in complicated surface imaging. In addition, it shows that $\bar{\epsilon}=0.081 \lambda$ for SEABED, $\bar{\epsilon}=0.097 \lambda$ for Envelope, and $\bar{\epsilon}=0.060 \lambda$ for the proposed method. This evaluation quantitatively proves the effectiveness of our proposed method. Furthermore, in the case of the target which depolarizes an incident wave such as conductive cylinder with length much greater than radius, the proposed method hardly reconstructs its original image because this depolarization highly suppresses the received signals, which reduces the estimation accuracy for $\left(x\left(\boldsymbol{q}^{3 d}\right), y\left(\boldsymbol{q}^{3 d}\right)\right)$ as in (23) and (24).

\section{CONCLUSION}

We have proposed a novel imaging algorithm without range point connections for complex shape targets. First, we dis- cussed the characteristic of the images estimated by the conventional algorithms as SAR, F-K migration, SEABED, Envelope, and IBST with Fourier transform. Next, we presented an accurate and high-speed imaging algorithm with the evaluation function of DOA. This algorithm does not require connections between range points and yet substantially resolves the inaccuracy of the conventional algorithm. The numerical simulations show that the proposed method increases substantially the number of estimated points with $\epsilon<0.1 \lambda$, and also the detection probability, even for the most complex shaped targets. It was also shown that the calculation time for the proposed method 2-D model is within $0.2 \mathrm{~s}$, which is appropriate for real-time operation.

We also extended the proposed algorithm to 3-D modeling and made statistical calculation for the $x$ and $y$ target coordinates. It was confirmed that this method accomplishes accurate imaging even for 3-D complex targets. The calculation time for this method is around $50 \mathrm{~s}$, and it is important in our future work to enhance the speed of the imaging. For simple target boundaries such as trapezoidal or spherical targets, there are advantages in using the conventional algorithm Envelope in terms of real-time and superresolution imaging. It is promising to select or combine appropriate algorithms for the assumed application. In addition, all the algorithms in this paper can be implemented on the same hardware or radar system, able to measure the received electric field for each antenna location. Future work includes an experimental study of the proposed method.

\section{APPENDIX \\ ProOF OF PROPOSITION 1}

Here, we utilize the following proposition, which has been proved in [16].

Proposition 2: If $\partial x / \partial X>0$ holds for all $(X, Z)$, each target boundary $(x, z)$ satisfies

$$
(x-X)^{2}+z^{2} \geq Z^{2}
$$

where an equal sign holds at only one point of $(X, Z)$. Here, the target point is defined as $\left(x\left(X_{i}\right), z\left(X_{i}\right)\right)$, which satisfies $x\left(X_{i}\right)=X_{i}-Z_{i} Z_{i X_{i}}$, and $z\left(X_{i}\right)=Z_{i} \sqrt{1-\left(Z_{i X_{i}}\right)^{2}}$, where $Z_{i X_{i}}=\partial Z_{i} / \partial X_{i}$. Substituting $\left(x\left(X_{i}\right), z\left(X_{i}\right)\right)$ to (27) gives

$$
Z_{i}^{2}+\left(X_{i}-X\right)^{2}-Z^{2}-2 Z_{i} Z_{i X_{i}}\left(X_{i}-X\right) \geq 0 .
$$

Contrarily, the derivative of $x_{p}\left(X_{i}\right)$ for $X_{i}$ is expressed as

$$
\frac{\partial x_{p}\left(X_{i}\right)}{\partial X_{i}}=\frac{Z_{i}^{2}+\left(X_{i}-X\right)^{2}-Z^{2}-2 Z_{i} Z_{i X_{i}}\left(X_{i}-X\right)}{2\left(X-X_{i}\right)^{2}} .
$$

From (28)

$$
\frac{\partial x_{p}\left(X_{i}\right)}{\partial X_{i}} \geq 0
$$

holds. Moreover, if $\partial x / \partial X>0$, then (7) gives

$$
\left(X-X_{i}\right)\left(x-x_{p}\left(X_{i}\right)\right) \geq 0 .
$$


Thus

$$
\left.\begin{array}{l}
x_{p}\left(X_{j}\right) \leq x_{p}\left(X_{i}\right) \leq x, \quad\left(X_{j} \leq X_{i} \leq X\right) \\
x \leq x_{p}\left(X_{i}\right) \leq x_{p}\left(X_{j}\right), \quad\left(X \leq X_{i} \leq X_{j}\right)
\end{array}\right\}
$$

is proved. For $\partial x / \partial X<0$, the following relationship also holds with the similar approach:

$$
\left.\begin{array}{l}
x_{p}\left(X_{j}\right) \leq x_{p}\left(X_{i}\right) \leq x, \quad\left(X \leq X_{i} \leq X_{j}\right) \\
x \leq x_{p}\left(X_{i}\right) \leq x_{p}\left(X_{j}\right), \quad\left(X_{j} \leq X_{i} \leq X\right)
\end{array}\right\} .
$$

Equations (32) and (33) correspond to the Proposition 1.

\section{REFERENCES}

[1] D. L. Mensa, G. Heidbreder, and G. Wade, "Aperture synthesis by object rotation in coherent imaging," IEEE Trans. Nucl. Sci., vol. NS-27, no. 2, pp. 989-998, Apr. 1980

[2] D. Liu, G. Kang, L. Li, Y. Chen, S. Vasudevan, W. Joines, Q. H. Liu, J. Krolik, and L. Carin, "Electromagnetic time-reversal imaging of a target in a cluttered environment," IEEE Trans. Antennas Propag., vol. 53, no. 9 , pp. 3058-3066, Sep. 2005.

[3] D. Liu, J. Krolik, and L. Carin, "Electromagnetic target detection in uncertain media: Time-reversal and minimum-variance algorithms," IEEE Trans. Geosci. Remote Sens., vol. 45, no. 4, pp. 934-944, Apr. 2007.

[4] J. Song, Q. H. Liu, P. Torrione, and L. Collins, "Two-dimensional and three dimensional NUFFT migration method for landmine detection using ground-penetrating radar," IEEE Trans. Geosci. Remote Sens., vol. 44, no. 6, pp. 1462-1469, Jun. 2006.

[5] F. Soldovieri, A. Brancaccio, G. Prisco, G. Leone, and R. Pieri, "A Kirchhoff-based shape reconstruction algorithm for the multimonostatic configuration: The realistic case of buried pipes," IEEE Trans. Geosci. Remote Sens., vol. 46, no. 10, pp. 3031-3038, Oct. 2008.

[6] R. H. Stolt, "Migration by Fourier transform," Geophysics, vol. 43, no. 1, pp. $23-48$, Feb. 1978

[7] X. Xu, E. L. Miller, and C. M. Rappaport, "Minimum entropy regularization in frequency-wavenumber migration to localize subsurface objects," IEEE Trans. Geosci. Remote Sens., vol. 41, no. 8, pp. 1804-1812, Aug. 2003.

[8] A. Massa, D. Franceschini, G. Franceschini, M. Pastorino, M. Raffetto, and M. Donelli, "Parallel GA-based approach for microwave imaging applications," IEEE Trans. Antennas Propag., vol. 53, no. 10, pp. 31183127 , Oct. 2005

[9] C. Chiu, C. Li, and W. Chan, "Image reconstruction of a buried conductor by the genetic algorithm," IEICE Trans. Electron., vol. E84-C, no. 12, pp. 1946-1951, 2001.

[10] T. Sato, T. Wakayama, and K. Takemura, "An imaging algorithm of objects embedded in a lossy dispersive medium for subsurface radar data processing," IEEE Trans. Geosci. Remote Sens., vol. 38, no. 1, pp. 296-303, Jan. 2000.

[11] Q. Zhang, T. S. Yeo, H. S. Tan, and Y. Luo, "Imaging of a moving target with rotating parts based on the Hough transform," IEEE Trans. Geosci. Remote Sens., vol. 46, no. 1, pp. 291-299, Jan. 2008.

[12] S. A. Greenhalgh, D. R. Pant, and C. R. A. Rao, "Effect of reflector shape on seismic amplitude and phase," Wave Motion, vol. 16, no. 4, pp. 307-322, Dec. 1992.

[13] T. Sakamoto and T. Sato, "A target shape estimation algorithm for pulse radar systems based on boundary scattering transform," IEICE Trans. Commun., vol. E87-B, no. 5, pp. 1357-1365, 2004.

[14] T. Sakamoto, "A fast algorithm for 3-dimensional imaging with UWB pulse radar systems," IEICE Trans. Commun., vol. E90-B, no. 3, pp. 636-644, 2007.

[15] S. A. Greenhalgh and L. Marescot, "Modeling and migration of 2-D georadar data: A stationary phase approach," IEEE Trans. Geosci. Remote Sens., vol. 44, no. 9, pp. 2421-2429, Sep. 2006.

[16] S. Kidera, T. Sakamoto, and T. Sato, "A robust and fast imaging algorithm with an envelope of circles for UWB pulse radars," IEICE Trans. Commun., vol. E90-B, no. 7, pp. 1801-1809, Jul. 2007.

[17] S. Kidera, T. Sakamoto, and T. Sato, "High-resolution and real-time UWB radar imaging algorithm with direct waveform compensations," IEEE Trans. Geosci. Remote Sens., vol. 46, no. 11, pp. 3503-3513, Nov. 2008.

[18] T. C. Williams, J. M. Sill, and E. C. Fear, "Breast surface estimation for radar-based breast imaging system," IEEE Trans. Biomed. Eng., vol. 44, no. 6, pp. 1678-1686, Jun. 2008.
[19] L. Capineri, L. Masotti, and S. Rocchi, "A 3D airborne ultrasound scanner," Meas. Sci. Technol., vol. 9, no. 6, pp. 967-975, Sep. 1998

[20] S. Hantscher, B. Etzlinger, A. Reisezahn, and C. G. Diskus, "A wave front extraction algorithm for high-resolution pulse based radar systems," in Proc. ICUWB, Sep. 2007, pp. 590-595.

[21] T. Sakamoto, H. Matsumoto, and T. Sato, "3-D fast imaging method for UWB radar in interference-rich environments with global optimization," Tech. Rep. IEICE, vol. 108, no. 318, pp. 129-134, Nov. 2008.

[22] C. G. Windsor and L. Capineri, "Automated object positioning from ground penetrating radar images," ISIGHT, J. British Inst. Non Destruct. Test., vol. 40, no. 7, pp. 482-488, Jul. 1998.

[23] S. Kidera, Y. Kani, T. Sakamoto, and T. Sato, "Fast and accurate 3-D imaging algorithm with linear array antennas for UWB pulse radars," IEICE Trans. Commun., vol. E91-B, no. 8, pp. 2683-2691, 2008.

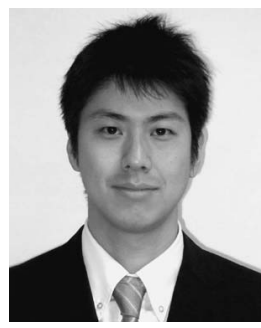

Shouhei Kidera (A'08) received the B.E. degree from Kyoto University, Kyoto, Japan, in 2003 and the M.I. and Ph.D. degrees from the Graduate School of Informatics, Kyoto University, in 2005 and 2007, respectively.

$\mathrm{He}$ is currently an Assistant Professor with the Department of Electronic Engineering, Graduate School of Electro-Communications, The University of Electro-Communications, Tokyo, Japan. His current research interest is in advanced signal processing for the near-field radar and ultrawide-band radar. Dr. Kidera is a member of the Institute of Electronics, Information, and Communication Engineers of Japan and the Institute of Electrical Engineering of Japan.

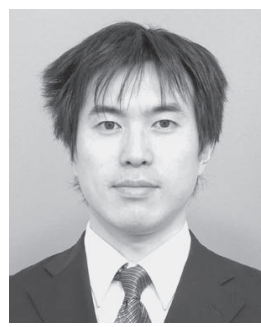

Takuya Sakamoto (M'04) was born in Nara, Japan, in 1977. He received the B.E. degree from Kyoto University, Kyoto, Japan, in 2000 and the M.I. and $\mathrm{Ph}$.D. degrees from the Graduate School of Informatics, Kyoto University, in 2002 and 2005, respectively.

$\mathrm{He}$ is currently an Assistant Professor with the Department of Communications and Computer Engineering, Graduate School of Informatics, Kyoto University. His current research interest is in signal processing for ultrawide-band pulse radars.

Dr. Sakamoto is a member of the Institute of Electronics, Information, and Communication Engineers of Japan and the Institute of Electrical Engineering of Japan.

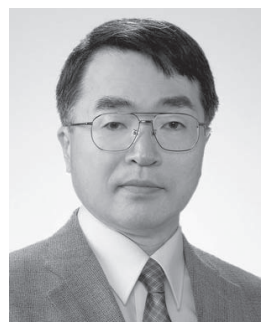

Toru Sato (M'92) received the B.E., M.E., and $\mathrm{Ph} . \mathrm{D}$. degrees in electrical engineering from Kyoto University, Kyoto, Japan, in 1976, 1978, and 1982, respectively.

Since 1983, he has been with Kyoto University, where he is currently a Professor with the Department of Communications and Computer Engineering, Graduate School of Informatics. His major research interests have been in system design and signal processing aspects of atmospheric radars, radar remote sensing of the atmosphere, observations of precipitation using radar and satellite signals, radar observation of space debris, and imaging with ultrawide-band pulse radars.

Dr. Sato is a Fellow of the Institute of Electronics, Information, and Communication Engineers of Japan and a member of the Society of Geomagnetism and Earth, Planetary and Space Sciences, the Japan Society for Aeronautical and Space Sciences, and the American Meteorological Society. He was the recipient of the Tanakadate Prize in 1986. 